\title{
Una metodología para la utilización didáctica del juego en contextos educativos
}

\author{
Maite Garaigordobil
}

\section{$\mathcal{M a}$ i}

Este artículo presenta la didáctica aplicada de un programa de intervención psicoeducativa, basado en los juegos de ayuda y cooperación, que tiene por finalidad estimular la socialización y la conducta prosocial en niños de 6 a 8 años (ciclo inicial). El trabajo analiza el valor del juego y de la interacción cooperativa entre iguales en el desarrollo infantil, describiendo un programa de juego, su fundamentación teórica, sus objetivos asi como las caracteristicas de los juegos que lo componen. Posteriormente, se expone el procedimiento metodológico para ser aplicado en el aula, asi como algunos instrumentos para su evaluación. La didáctica diseñada y propuesta en esta intervención, tiene la ventaja de ser generalizable en la aplicación de programas de juego psicomotriz, creativo..., que estimulen otras áreas del desarrollo infantil. Además, este programa de juego que se ha venido aplicando desde el año 1989 en un conjunto de aulas de la provincia de Guipuzcoa, ha sido validado mediante una investigación que evidenció un efecto positivo de esta intervención en distintas variables del desarrollo infantil.

\section{INTRODUCCION: MARCO TEORICO-CONCEPTUAL DEL PROGRAMA}

La intervención psicoeducativa que proponemos tiene una perspectiva ecléctica, ya que se fundamenta teóricamente en las conclusiones obtenidas desde tres líneas de investigación. Por un lado, la que se deriva de los estudios que han clarificado las relaciones entre juego y desarrollo, por otro lado, la que proviene de los análisis realizados sobre la importancia de la interacción entre iguales, y finalmente, la de los trabajos que han evidenciado los positivos efectos de cooperar versus competir en el contexto escolar.

Contribuciones del juego al desarrollo integral

Los datos provenientes de numerosos estudios, planteados desde distintos marcos epistemológicos, permiten concluir que el juego, esa actividad por excelencia de la infancia, contribuye de forma relevante al desarrollo integral del niño (Garaigordobil, 1990). En la actualidad se ha comprobado que el juego desempeña un papel importante en el desarrollo intelectual, ya que a través de las variadas activida- 
des lúdicas que realiza el niño a lo largo de la infancia, crea y desarrolla estructuras mentales (Piaget, 1959/1979; Kamii y Devries, 1980/1988), que posibilitan una vía de desarrollo del pensamiento abstracto (Vygotski, 1933/1982; Piaget e Inhelder, 1969/1984; Elkonin, 1978/1980), ensaya conductas más complejas (Ortega, 1986), siendo un estímulo para la atención, y la memoria (Mujina, 1975). Además el juego fomenta el descentramiento egocéntrico (Piaget, 1959/1979), promueve la creatividad y la imaginación del niño (Vygotski, 1933/1982; Bruner, 1986) desempeñando una función muy positiva en el desarrollo del lenguaje (Zabalza, 1987; Fisher, 1992; Levy y otros, 1992; Pellegrini y Galda, 1993).

En lo referente al desarrollo social, también es un hecho confirmado que las actividades lúdicas son un relevante instrumento de comunicación y socialización (Ortega, 1987; 1991a.b), ya que cuando los niños representan el mundo del adulto, descubren la vida social de los adultos y las reglas por las que se rigen estas relaciones, aprenden los derechos y los deberes de cada rol, así como, distintas funciones sociales, preparándose para el trabajo (Mujina, 1975). Además, en el juego interactúa con otros compañeros, lo que le permite ampliar sus formas de comunicación, desarrollar su capacidad de cooperación (Kamii y Devries, 1980/1988), y sus habilidades sociales (McCune-Nicolish, 1981). Otra función socializadora del juego se deriva de ser un estímulo para el desarrollo moral, ya que diversos estudios han evidenciado que esta actividad es escuela de autodominio, de voluntad, y de asimilación de normas de conducta (Elkonin, 1978/1980; Pardos y otros, 1988).

Por otro lado, los trabajos de enfoque psicoanalítico, enfatizan otra importante contribución de la actividad lúdica infantil al desarrollo afectivo-emocional (Winnicott, 1971/1982). Como resultado de sus observaciones se constata que el juego es una fuente de placer que estimula la alegría de vivir, además de una vía de liberación de la ansiedad que se deriva de fuentes externas (experiencias difíciles en la realidad) e internas (sexualidad-agresividad). Esta función del juego como instrumento de expresión emocional y de descarga de tensiones, hace que esta actividad promueva el equilibrio psíquico y la salud mental.

\section{El papel de la interacción entre iguales en el desarrollo cognitivo y socio- afectivo}

La segunda línea de investigación sobre la que se sustenta el programa, es la de los estudios que han confirmado el papel de la interacción entre iguales en variados procesos del desarrollo cognitivo y socio-afectivo. Algunos de estos trabajos se han operado desde un enfoque cognitivo-evolutivo, concluyendo que este tipo de interacción contribuye positivamente al desarrollo cognitivo-moral. Estas investigaciones subrayan que cuando los niños interactúan con otros en la realización de una actividad grupal, surgen conflictos originados por su egocentrismo. En opinión de Piaget (1932/1974), estos conflictos provocan reestructuraciones cognitivas internas, que promueven el desarrollo intelectual, siendo también, este tipo de interacción fuente de desarrollo moral, ya que potencia el desarrollo de la moralidad autónoma. Además, en esta situación grupal de discusión y debate los niños desarrollan el proceso de adopción de perspectivas, a partir del cual se construye el conocimiento de sí mismo y el de los demás (Kohlberg, 1982).

Los enfoques etológicos y de observación naturalista han visto la interacción entre iguales como una fuente de experiencias afectivas que influyen en la adaptación socio-emocional. Desde esta perspectiva, evidencian el significativo papel de esta interacción, en el desarrollo de conductas adaptadas de expresión emocional, en el control de la agresión, y en el aprendizaje de conductas sexuales adecuadas, observándose a través de diversos estudios, que la privación temprana de estas interaccio- 
nes, genera problemas de timidez, retraimiento o conducta agresiva (Blurton-Jones, 1967).

Desde otro encuadre teórico, neoconductistas y teóricos del aprendizaje, enfatizan que el grupo de iguales es un modelo de conducta, y un agente de refuerzos, que resulta de gran eficacia para la adquisición y mantenimiento tanto de conductas deseables, como indeseables, siendo además, una fuente de influencia en la percepción de la propia autoeficacia (Hoffman, 1983), que afecta tanto a los procesos de pensamiento como a las reacciones emocionales. Finalmente, los psicólogos soviéticos proponen que la comunicación en una situación cooperativa entre compañeros actúa como contexto intermedio en la interiorización de los procesos psicológicos superiores. Consideran el lenguaje como el nudo central del proceso de interiorización, que tiene por motivación la necesidad de comunicar a los compañeros la propia representación. Ello a su vez da lugar a la revisión del propio punto de vista en relación a otras perspectivas comunicadas por los otros. También, desde este punto de vista, la posición que se ocupa dentro del grupo de iguales es una importante motivación para la actividad escolar, e influye de forma decisiva en la autovaloración y en la adaptación al sistema educativo (Vygotski, 1933/1982; 1934/1979; Forman y Cazden, 1984).

\section{Los beneficios de jugar y aprender cooperativamente en el contexto escolar}

La tercera línea de investigación que subyace al programa proviene de los trabajos que han verificado los beneficios individuales y grupales de cooperar versus competir en el contexto escolar. Desde estas investigaciones, se ha puesto de manifiesto que los niños interactuando cooperativamente, mantienen comunicaciones más eficaces, verbalizan más ideas, aceptan más las ideas de los otros, mostrando mayor coordinación, esfuerzo y productividad cuando realizan una tarea (Deutsch, 1971). También se ha verificado que la actividad cooperativa facilita la reducción de conflictos intergrupos (Sherif, y otros, 1961), favorece el establecimiento de relaciones más positivas entre los compañeros, como aquellas caracterizadas por la atención, la simpatía, la cortesía y el respeto mutuo (Coll, 1984). Además, el ambiente cooperativo mejora el clima del aula y las relaciones interpersonales en el seno del grupo (Rué, 1989), posibilitando el marco más idóneo para el desarrollo de unas adecuadas habilidades sociales (Ovejero, 1990; 1993).

Aunque son pocos los estudios que han analizado las relaciones entre juego cooperativo y desarrollo infantil, sin embargo, los que han abordado estas vinculaciones concluyen que este tipo de juego promueve un incremento de las conductas de compartir (Orlick, 1981; 1988), y de las respuestas sociales cooperativas en niños con dificultades de desarrollo (Mender y otros 1982). Mucho más fecunda ha sido la investigación con distintas técnicas de aprendizaje cooperativo, existiendo un gran cúmulo de resultados que evidencian la eficacia del aprendizaje cooperativo en el desarrollo académico (Jhonson y Jhonson, 1986; Slavin, 1983; 1990), en la memoria (Hall, y otros, 1988), y en la motivación intrínseca frente a las tareas académicas (Butler, 1989). De todos modos, tanto el juego como el aprendizaje cooperativo se sustentan en principios teóricos interaccionistas, desde los que se considera que la inteligencia, la motivación intrínseca, o el autoconcepto son todos ellos construidos socialmente, en el transcurso de las interacciones sociales cotidianas.

Aunque con el programa de juego intentamos desarrollar conductas de ayuda y cooperación entre los niños, no obstante, somos conscientes de que la conducta prosocial es una conducta compleja (Sterling, 1990) y que en ella influyen múltiples factores como la cultura, la educación parental, el contexto escolar, o la personalidad (desarrollo cognitivo, moral, de la capacidad de toma de perspectiva y de la empatía). Sin embargo, y pese a la complejidad de este tipo de conducta, influida por 
numerosas variables, las experiencias realizadas permiten constatar la eficacia de programas de entrenamiento para la adquisición de pautas de comportamiento altruista en los niños (Cascón y Martín, 1986; Masnou, 1991; Ortíz, y otros, 1991; De la Cruz y Mazaira, 1992).

\section{LA INVESTIGACION DEL PROGRAMA}

Esta propuesta didáctica se fundamenta en una investigación realizada previamente que ha sido presentada como tesis doctoral (Garaigordobil, 1992a), en la que se evaluó el efecto del programa de juego en distintos aspectos del desarrollo del niño y en las relaciones intragrupo en el contexto escolar. El estudio empleó un diseño pretest-intervención-postest que se llevó a cabo con una muestra de 178 niños de $1^{\circ}$ de EGB, distribuidos en 8 aulas públicas y privadas, de la provincia de Guipuzcoa. Del conjunto de la muestra, 125 niños (6 grupos) desempeñaron la condición experimental, y 53 ( 2 grupos) la de control. El procedimiento se realizó en tres fases: al comienzo del curso escolar se aplicaron diversos instrumentos de evaluación para medir algunos aspectos del desarrollo (ver Tabla 4), posteriormente, los grupos experimentales realizaron el programa de juego durante un curso escolar (1 sesión de juego semanal), y al finalizar éste se operó de nuevo la misma evaluación.

Después de realizar distintos análisis estadísticos (MANOVA, CLUSTER, ANOVA) y observacionales con los datos obtenidos en la evaluación, se puso de manifiesto que los niños que realizaron el programa de juego versus los de control, incrementaron significativamente en el contexto del aula sus conductas de liderazgo-iniciativa, de jovialidad, de sensibilidad social, y de respeto por las normas de la sociabilidad-autocontrol de los impulsos, así como las conductas de adaptación social global, disminuyendo sus conductas agresivas, de apatía-retraimiento y de ansiedad-timidez. También la conducta social con los compañeros en el contexto familiar, extraescolar, mejoró significativamente, pero en menor medida y únicamente en las conductas de liderazgo-iniciativa, de sensibilidad social, de adaptación social global, y en las conductas agresivas. Por otro lado, se confirmó una repercusión positiva del programa en las aptitudes relacionadas con la madurez para el aprendizaje escolar (comprensión verbal, aptitudes numéricas), en el esquema corporal, en la cantidad y calidad de las estrategias cognitivas de interacción social disponibles por el niño y en el autoconcepto.

La observación cualitativa realizada con la información sociométrica, verificó mayores relaciones de aceptación mutua en las interacciones intragrupo en el aula, y una mejora de la capacidad de cooperación grupal al evidenciarse que el programa había potenciado una disminución del tiempo de ejecución necesario para realizar una tarea cooperativa, y un incremento de conductas facilitadoras de la cooperación como «Dar-Recibir» «Pedir-Recibir»y «Ayudar con indicaciones o manipulaciones».

Los resultados obtenidos en nuestro estudio (Garaigordobil, 1992a.b; 1993) ratifican las observaciones e investigaciones que subrayan el positivo papel de la interacción lúdica y la cooperación en el desarrollo infantil (Orlick, 1981; Martínez, 1986; Ovejero, 1990; 1993). La aplicación de este programa de juego en diversas aulas del ciclo inicial durante 4 años, así como la investigación realizada con él, ratifica la trascendencia del juego amistoso-prosocial en el desarrollo del niño y en las relaciones sociafectivas y de cooperación en el grupo escolar. Este trabajo que varios grupos de profesionales hemos venido realizando con juegos amistosos, de ayuda y cooperación nos ha permitido, por un lado, confirmar que el juego que es acción, comunicación y cooperación desempeña un papel importante en la socialización 
infantil, y por otro, sistematizar una metodología de utilización del juego en la intervención psicoeducativa, que es generalizable a la aplicación de programas de juego psicomotriz, creativo.... para estimular otras dimensiones del desarrollo infantil.

Además, esta experiencia tiene implicaciones prácricas para la integración grupal de niños con dificultades de desarrollo, habiéndose confirmado que el programa fue especialmente beneficioso para los niños que en la fase pretest mostraban bajo autoconcepto, y muchas conductas sociales de apatía-retraimiento y ansiedadtimidez con sus iguales.

\section{EL PROGRAMA DE JUEGO: DESCRIPCION Y PROCEDIMIENTO DIDACTICO}

\section{Objetivos del programa}

La intervención tiene por objetivo principal estimular el desarrollo en los niños que evolucionan con normalidad, especialmente en su dimensión socio-afectiva. No obstante, incluye una perspectiva terapéurica, ya que la intervención fomenta la integración grupal de niños con dificultades de desarrollo (inmaduros para el aprendizaje, bajo autoconcepto...) y en particular, la integración de niños con problemas en la socialización (tímidos, retraídos, aislados, agresivos-impositivos...). De forma específica, las actividades lúdicas que componen el programa pretenden: a) Ampliar el conocimiento de los niños entre sí; b) Potenciar la interacción multidireccional, positiva, amistosa, constructiva, y la participación grupal; c) Incrementar conductas sociales facilitadoras de la socialización (conductas de liderazgo, de jovialidad, de sensibilidad social, de respero-autocontrol), así como disminuir las conductas perturbadoras (conductas agresivas, de apatía-retraimiento, de ansiedad-timidez); d) Mejorar las habilidades de comunicación, los hábitos de escucha activa; e) Estimular relaciones de ayuda y de cooperación intragrupo; f) Promover la expresión integral de la personalidad; y g) Mejorar la autoimagen.

\section{Características y clasificación de los juegos del programa}

Los juegos que componen el programa tienen en su base la idea de aceptarse, cooperar y compartir, estando caracterizados por cinco componentes estructurales: a) La «Acción y Participación», ya que en estos juegos todos participan, nadie sobra, nadie queda eliminado, ni pierde, con lo que se estructura un sentimiento de pertenencia al grupo en cada individuo; b) La "Cooperación", porque la dinámica de estos juegos estimula una interacción amistosa multidireccional positiva intragrupo, para progresivamente porenciar procesos en los que los jugadores dan y reciben ayuda para contribuir a un fin común. En los juegos cooperativos los objetivos de los jugadores están estrechamente vinculados, y los resultados que persigue cada miembro del grupo, son beneficiosos para el resto de los compañeros con los que está interactuando, en contraposición a los juegos competitivos en los que los objetivos de los jugadores están relacionados pero de forma excluyente, y un participante alcanza la meta, sólo sí los otros no la consiguen alcanzar; c) La «Aceptación», porque en estas actividades lúdicas cada jugador contribuye con su papel, y éste papel, lleno de significado, es necesario para la consecución del juego. Esta situación genera un sentimiento de aceptación en cada individuo del grupo, que influye positivamente en su propia imagen; d) La «Ficción», ya que en la mayor parte de los juegos del programa se encuentra presente el hacer el "como si» de la realidad (convirtiéndose los jugadores sucesivamente en serpientes, campanas, trenes, ciegos, máquinas....); $\epsilon$ ) La 
«Diversión», porque con estos juegos pretendemos que los jugadores se diviertan interactuando de forma positiva y constructiva con sus compañeros.

Después de revisar más de 1.500 actividades lúdicas se seleccionaron los 60 juegos que componen el programa, algunos de los cuales son actividades lúdicas de las pioneras experiencias con juegos de cooperación de Orlick (1978/1986), otros son juegos tradicionales, y otros, trasformaciones de juegos competitivos en cooperativos mediante la modificación de sus reglas. Para la selección de los juegos se tomó como criterio la estructura básica del juego definida por los cinco componentes o características que se han descrito previamente, siendo estos componentes requisitos para su incorporación al programa. Además, los juegos incluidos en el programa fueron agrupados en 6 niveles o bloques (ver tabla I) en base a un análisis estructural que se realizó de cada juego (ver tabla II). Para operar esta clasificación se tomó como criterio el objetivo principal o aspecto básico del desarrollo que cada juego promueve (comunicación intragrupo, relaciones de ayuda, cooperación, etc).

Dentro del primer nivel, se incluyen tres «Juegos de Presentación», que inician las primeras sesiones de juego, y tienen por objerivo facilitar el conocimiento inicial de los miembros del grupo entre sí. Un ejemplo de este tipo de juego es «Tarjeta de Visita», en el que cada jugador elabora una tarjeta de presentación, escribiendo su nombre en letras grandes sobre un folio en el que dibuja algo que le represente (si le gusta el monte puede hacer un paisaje, si le gusta leer, un libro....). Posteriormente, se ponen en círculo y cada participante mostrando su tarjeta, se presenta a los demás.

El segundo nivel o bloque, "Juegos de comunicación verbal y no verbal» se compone de trece actividades lúdicas que tienen por finalidad estimular la comunicación intragrupo, y el desarrollo de los hábitos de escucha activa. Los juegos que implican formas de comunicación verbal y no verbal, suponen innumerables experiencias para la estimulación de las relaciones interpersonales y el fortalecimiento del grupo, favoreciendo nuevas posibilidades de comunicación y unas relaciones más cercanas y abiertas. Un ejemplo de este tipo de juegos es «Palabras encadenadas», en el que los jugadores, por turnos, deben construir una frase que comience con la última palabra de la frase verbalizada por el compañero anterior, o «El corral» en el que los jugadores se distribuyen por parejas cada una de las cuales representará un animal. Posteriormente, con los ojos cerrados y caminando a cuatro patas deben encontrar a su compañero, utilizando para ello la emisión del sonido específico del animal.

El tercer nivel, «Juegos de dar y recibir ayuda» está constituido por siete juegos que generan situaciones relacionales en las que los niños dan ayuda y reciben ayuda porque la dinámica de la actividad lúdica propone este tipo de acción para poder seguir jugando. Con estos juegos se pretende estimular que los niños observen las necesidades de otros y respondan positivamente a las mismas, mejorando tanto las relaciones de ayuda entre los miembros del grupo, como la imagen del «Yo» en cada uno de ellos. Un juego de este tipo es el «Stop», en el que un jugador elegido por sorteo representa al perseguidor y tiene que tocar a sus compañeros, mientras éstos corren tratando de evitar ser alcanzados. Cuando el perseguidor toca a algún niño, le dice «Stop» y éste queda paralizado, con las piemas y brazos abiertos. Otro compañero deberá pasar arrastrándose por debajo de las piernas del paralizado para librarle de la inmovilidad, tarea nada fácil, ya que el perseguidor intentará tocarle y dejarle paralizado también. Otra actividad de este bloque es «Zapatos Viajeros». Ahora los niños depositan uno de sus zapatos en una montaña de zapatos que se sitúa en el centro del círculo, y a la orden del director de juego (profesor/a) cada jugador debe coger el zapato de otro compañero, buscar a su dueño y dárselo; y todo ello a gran velocidad. 


\begin{tabular}{|c|c|c|c|}
\hline \multirow{7}{*}{ 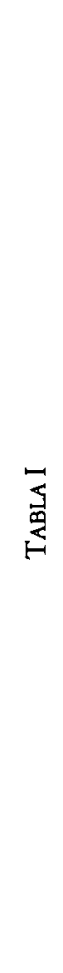 } & NIVEL DE JUEGO & OBJETIVOS & LISTADO DE ACTIVIDADES \\
\hline & Juegos de Presentación & $\begin{array}{l}\text { * Conocimiento inicial del grupo } \\
\text { * Autoafirmación personal }\end{array}$ & Este es mi amigo. El gesto. Tarjeca de Visita. \\
\hline & $\begin{array}{l}\text { Juegos de } \\
\text { Comunicacion } \\
\text { intragrupo }\end{array}$ & $\begin{array}{l}\text { * Comunicación intragrupo positiva } \\
\text { * Comunicación Verbal y no Verbal } \\
\text { * Hábitos de escucha activa } \\
\text { * Mejora autoconcepto }\end{array}$ & $\begin{array}{l}\text { El telegrama. Abrazos Musicales. El Chicle. Controla tu } \\
\text { risa. } \\
\text { Los Espejos. El corral. E1 Disparate. Simón dice. } \\
\text { Las cosas buenas de mis compañeros. La Estatua. } \\
\text { Los complementos. Palabras encadenadas. Los despropositos }\end{array}$ \\
\hline & $\begin{array}{l}\text { Juegos de Dar y } \\
\text { Recibir ayuda }\end{array}$ & $\begin{array}{l}\text { * Observar las necesidades de otros } \\
\text { * Conductas de ayuda a compañeros }\end{array}$ & $\begin{array}{l}\text { Los saquitos de arena descongelan. Pelota a Dos. El muro. } \\
\text { Levantar al compañero. Subir de espaldas. Zapatos viajeros. } \\
\text { Stop. }\end{array}$ \\
\hline & Juegos de Confianza & * Confianza en los demás & $\begin{array}{l}\text { El Ciego. La alfombra voladora. El tren. Círculo de amigos. } \\
\text { Plo-Pfo. Crí-Crr, Trás-Trás,.... La muralla. }\end{array}$ \\
\hline & Juegos de Cooperación & $\begin{array}{l}\text { * Comunicación Verbal-No verbal } \\
\text { * Toma de decisiones por consenso } \\
\text { * Observar necesidades de otros } \\
\text { * Dar y recibir ayuda } \\
\text { * Contribuir a fines comunes }\end{array}$ & $\begin{array}{l}\text { Las Ruedas. Que el globo no caiga al suelo. Las campanas. } \\
\text { La serpiente. Sillas no eliminatorias. Los Aros musicales. } \\
\text { Bolos en cooperación. Las Tortugas. Puzzles y } \\
\text { rompecabezas cooperativos. Construcción-Modelado } \\
\text { cooperativo. La Maquina. El lavacoches. Tubos rodantes. La } \\
\text { Orquesta. Una historia entre varios. Carreras de relevos. } \\
\text { Dactilopintura cooperativa. Dibuja una canción con rus } \\
\text { amigos. Bazar de palabras. El juego de los cuadrados. En } \\
\text { busca del tesoro. }\end{array}$ \\
\hline & $\begin{array}{l}\text { Juegos cooperativos de } \\
\text { expresión dramática }\end{array}$ & $\begin{array}{l}\text { * Comunicación intragrupo } \\
\text { * Interacción cooperativa } \\
\text { * Expresión emocional } \\
\text { * Expresión integral de la personalidad }\end{array}$ & $\begin{array}{l}\text { Sirve para todo. La cadena. La Selva. El accidente. } \\
\text { Emisión Radiofónica. Libre improvisación. El tribunal. } \\
\text { J. Dramático semiestructurado. J. Dramático libre. }\end{array}$ \\
\hline
\end{tabular}

|Nota: La descripción de las actividades lúdicas se presenta en la obra "Juego cooperativo y socializacion en el aula" (Garaigordobil, 1992.b) 


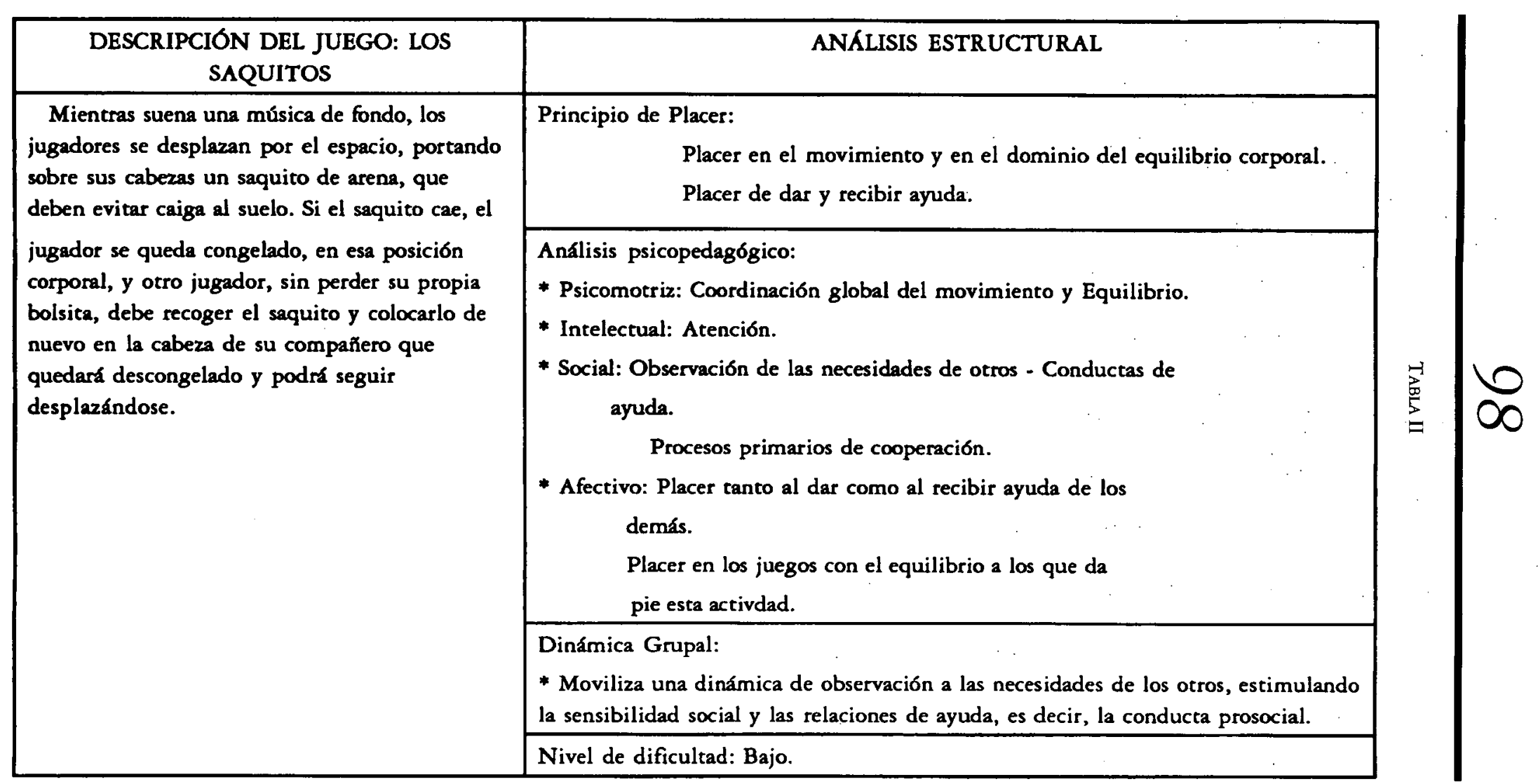


El cuarto nivel, "Juegos de confianza», está formado por siete actividades lúdicas que estimulan la confianza en los demás, ya que la confianza en el grupo es un requisito para que se den las actitudes solidarias necesarias, que permitan posteriormente procesos de cooperación grupal. Un juego de este bloque es el «El tren», en el que los jugadores se dividen en pequeños grupos de cuatro ó cinco niños, y cada grupo simboliza un tren que se construye al situarse un niño detrás de otro, en fila y colocando los brazos sobre los hombros del que va delante. Todos los vagones van ciegos (ojos cerrados), salvo los maquinistas del tren, que van los últimos en la fila. Antes de comenzar el juego, todos los jugadores conocen el código que el conductor trasmitirá no verbalmente para conducir el tren (Un golpe sobre la cabeza = Adelante; Un golpe en el hombro izquierdo = Girar a la izquierda...). Los trenes se ponen en situación de salida y el maquinista comienza a trasmitir los mensajes que movilizan los desplazamientos de los trenes.

El quinto nivel, «Juegos de cooperación», es el de mayor contenido, y en él se incluyen veintiún juegos que potencian situaciones relacionales, en las cuales los miembros del grupo se ayudan para contribuir a un fin común. Son juegos en los que la colaboración entre los jugadores es un elemento esencial para la realización de la actividad, ya que sin ella no es posible llegar a la meta del juego. Un ejemplo de este tipo de juegos es «Bazar de Palabras». En esta actividad los jugadores se dividen en grupos de 5 y se les propone como objetivo construir cinco frases, utilizando las palabras que tienen contenidas en los cinco sobres (uno por jugador). Para ello deben intercambiar palabras, cediendo y recibiendo palabras, pero deben hacerlo sin hablar durante el juego. Este bloque también incluye juegos de cooperación corporal, tales como «Las serpientes» o "Las tortugas» en los que se forman con los cuerpos de varios jugadores, serpientes que reptan por la selva, o tortugas que se desplazan por la arena. Otro grupo de actividades cooperativas son las constructivas, en las que se construye, modela o monta un puzzle mediante procesos de comunicación y estrategias cooperativas en las que los jugadores comparten sus piezas, distribuidas igualitariamente, para construir un castillo, modelar una escultura o reconstruir un puzzle o un rompecabezas.

En el último nivel, «Juegos cooperativos de expresión dramática», se incluyen nueve actividades lúdicas que promueven la interacción cooperativa y la ficción dramática posibilitando una vía de autoexpresión integral de la personalidad infantil, a través de la ficción dramática. En estos juegos las emociones encuentran un canal de expresión y liberación, además de ser un medio de comunicación con los demás. Aquí se propone el juego dramático libre, en el que los niños distribuidos en pequeños grupos inventan una historia, distribuyen los roles, construyen los materiales que requieren y realizan la representación para el resto de los compañeros. Además, se han incorporado a este nivel otros juegos dramáticos más estructurados como «Sirve para todo", en el que los jugadores se sientan en círculo y en el centro se coloca un objeto, con el que deben ensayar simbólicamente todos los usos posibles que les sugiere su forma. Una pala de madera será sucesivamente, un palo de golf, una cuchara o un remo.

\section{Procedimiento para la aplicación del programa en el aula}

Para la aplicación del programa en el aula se mantienen metodológicamente 6 factores $o$ variables que permanecen constantes:

1) El número de sesiones anuales que nunca es inferior a 22 , aproximadamente 1 sesión semanal, es decir, 44 sesiones en los dos cursos de ciclo inicial.

2) El tiempo de duración de cada sesión que oscila entre 60 y 90 minutos en los que se realizan 4 o 5 actividades lúdicas. 
3) El espacio de aplicación del programa ya que siempre se realiza en el mismo espacio físico, generalmente el aula de psicomotricidad o el gimnasio.

4) Los adultos que desarrollan la experiencia, por un lado, el que realiza la intervención que es el profesor/a habitual de ese grupo, y, por otro lado, el observa$\mathrm{dor} / \mathrm{ra}$ que filma y construye un diario, registrando narrativamente lo que acontece en las sesiones.

5) La dinámica estructural de cada sesión de juego, que se organiza en torno a tres momentos o fases diferenciadas: apertura, desarrollo y cierre. Habitualmente la sesión se inicia con todos los niños sentados en posición circular donde se recuerda brevemente las ideas básicas sobre la finalidad de las sesiones de juego cooperativo (hacer amigos, ayudar, cooperar, divertirse...), y se procede a dar la consigna del primer juego que se va a realizar. Después de jugarlo los niños retornan al círculo y en esta situación, que procura relajación momentánea de la excitación creada por la actividad previa, se les explica la consigna del siguiente juego, y así sucesivamente se realizan 4 o 5 actividades. Al finalizar la secuencia de juego, se opera la fase de cierre. En esta fase los niños durante 10-15 minutos reflexionan sobre lo sucedido en la sesión, expresando aspectos significativos de la experiencia vivida, tanto en su vertiente positiva (placer, nuevos amigos...) como en la negativa (rechazos, no ayuda, transgresiones de normas...). Se potencia de esta forma la verbalización de las interacciones sociales experimentadas, los conflictos dimanantes de las mismas, así como, las estrategias de resolución empleadas para resolverlos. Por ello, esta fase tiene un aspecto metacognitivo de gran interés.

6) El procedimiento que el profesor/a y el observador/a siguen para planificar las sesiones. Este es un programa semiestructurado en el que se aporta una batería de juegos, una metodología para el análisis de los mismos y un procedimiento para planificar y aplicar la sesión. Ello tiene por objetivo potenciar que el profesor/a diseñe cada sesión fundamentándose en los criterios metodológicos generales y en la observación de la realidad específica de su grupo, es decir, desde una perspectiva de investigación sobre la propia acción. La planificación de la sesión requiere seleccionar las actividades, y posteriormente, secuenciarlas. Para seleccionar los juegos se realiza un doble análisis. Por un lado, un análisis estructural del juego (ver tabla II), del cual se deja constancia escrita, valorando 4 ítems en esta reflexión: (a) el grado y la naturaleza del placer del juego; (b) los aspectos del desarrollo psicomotriz, intelectual, afectivo o social que ese juego moviliza; (c) la dinámica relacional que genera entre los jugadores; (d) el nivel de dificultad.

Paralelamente, se realiza un análisis evolutivo valorando la edad de los niños con los que opera la experiencia y su nivel de desarrollo grupal. Después de estos análisis, se procede a organizar la secuencia de juego, ya que ésta influye en el proceso socio-afectivo de la sesión, en su clima. Para ello se tienen en cuenta una serie de sugerencias generales, tales como la combinación de actividades con alto nivel de acción corporal, con otras más relajadas o de mayor implicación cognitiva, o la combinación de distintas modalidades relacionales (pareja, pequeño grupo, gran grupo) en el transcurso de la sesión.

\section{Instrumentos de evaluación del programa}

La evaluación de los efectos del programa se lleva a cabo desde tres perspectivas: Una evaluación continua, una evaluación experimental pretest-postest, y una valoración postintervención.

En primer lugar, y con la finalidad de realizar una evaluación continua del impacto del programa, se construye un «Diario" mediante una técnica narrativa con la que registra lo que acontece en cada sesión. En este diario el observador/a describe lo sucedido en cada juego, así como las verbalizaciones de los niños en la fase de cie- 
rre, y tiene por objetivo dejar constancia de las interacciones y la dinámica lúdicogrupal que se evidencian en la sesión. Por ejemplo, si observamos el juego «Crí-Crí, Trás-Trás,...», en el que un jugador tiene que guiar los desplazamientos por el aula de otro compañero ciego, utilizando únicamente el sonido onomatopéyico que hayan acordado previamente, se podría registrar: «En general permanecen muy atentos a la conducción del otro, evitando, a través de las indicaciones del sonido, que los compañeros se choquen o golpeen con obstáculos o con los jugadores que van ciegos... a excepción de "X» que se aleja a una gran distancia del otro, y deja de emitir el sonido acordado con su compañero ciego, provocando que éste se pierda por el espacio del aula y choque con otros, ante lo que "X» se ríe manifiestamente.»

Además, habitualmente las sesiones son grabadas en video y posteriormente visionadas por los adultos que aplican el programa. Este visionado permite incorporar datos complementarios al diario, así como evaluar la sesión de juego, ya que al finalizar el mismo, el profesor/ra y el observador/ra valoran la sesión con un cuestionario de evaluación de las interacciones lúdico-grupales diseñado con esta finalidad (ver tabla III). Con este instrumento se puntúa mediante una escala de estimación, el modo como los niños acogen las actividades, la participación, el clima del grupo, las interacciones observadas, el acatamiento de las normas de los juegos, y la flexibilidad en los agrupamientos espontáneos.

TABLA III

\begin{tabular}{|c|c|c|c|c|c|c|c|c|c|c|}
\hline INDICADORES & 1 & 2 & 3 & 4 & s & 6 & 7 & 8 & 9 & 10 \\
\hline GRADO DE PLACI & & & & & & & & & & \\
\hline PARTICIPACIÓN & & & & & & & & & & \\
\hline $\begin{array}{l}\text { CLIMA DEL GRUY } \\
\text { Organizado } \\
\text { Alegre } \\
\text { Pacifico }\end{array}$ & & & & & & & & & & \\
\hline $\begin{array}{l}\text { INTERACCIONES } \\
\text { Amisrosas } \\
\text { Asociación flexible } \\
\text { Ayuda } \\
\text { Escucha } \\
\text { Cooperación } \\
\text { No conflictivas }\end{array}$ & & & & & & & & & & \\
\hline $\begin{array}{l}\text { ACATAMIENTO } \\
\text { REGLAS }\end{array}$ & & & & & & & & & & \\
\hline
\end{tabular}

En segundo lugar, y con el objerivo de valorar el nivel de cambio en diversos factores de desarrollo (conducta social, autoconcepto...) que se estimulan con estos juegos, se realiza una evaluación experimental, aplicando varios instrumentos de evaluación, antes y después de aplicar el programa. Generalmente se administran 20 3 instrumentos que cada profesor/a selecciona de la batería de 9 que se emplearon en la investigación (ver tabla IV).

Finalmente, al terminar el curso escolar se opera una valoración postintervención, ya que los niños cumplimentan un cuestionario (Garaigordobil, 1992b) que recoge su opinión de la experiencia. Con este instrumento informan sobre lo que les ha gustado más o menos, y valoran el nivel de cambio (algo, nada, bastante, mucho) que experimentan en ellos mismos antes y después del programa, en factores como comunicación con los compañeros del grupo, amistad, relaciones de ayuda, confianza, cooperación, expresión emocional, autoimagen... 


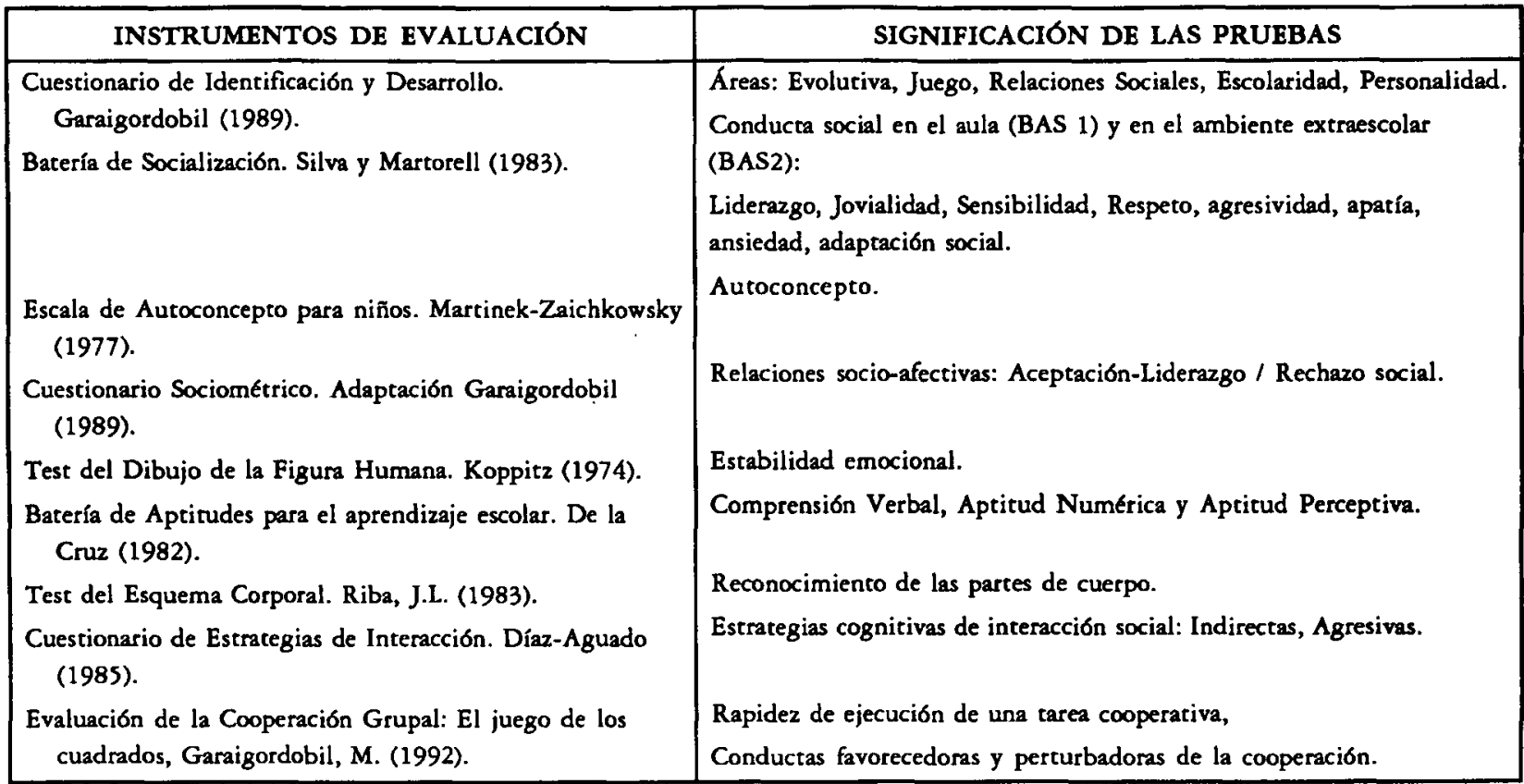

Nota: Si se utiliza el juego de los cuadrados como técnica de evaluación, no se incluye la actividad en la intervención. 
en los últimos años, hay argumentos para concluir que el juego cooperativo estimula positivamente el desarrollo social y afectivo, incrementando variadas conductas sociales facilitadoras de la socialización y elevando la autoestima. Constatado el papel que juegan los factores socio-afectivos en el desarrollo cognitivo y en el rendimiento académico, podemos considerar que este tipo de experiencias articuladas de forma sistemática tienen una gran potencialidad didáctica.

Este programa adaptado para niños de ciclo inicial es solo un primer paso en la construcción de una perspectiva psicosocial del trabajo educativo, que considera el grupo aula como un sistema de relaciones socio-afectivas, y que tiene por finalidad estimular las relaciones cooperativas en el contexto escolar. La aplicación del programa durante un curso escolar provee beneficios para los niños, sin embargo, creemos que este tipo intervención cobra pleno sentido cuando los niños tienen posibilidad de jugar o aprender cooperativamente en los sucesivos cursos académicos, para lo que se hace necesario diseñar propuestas educativas adaptadas a niños de distintas edades, es decir, para los distintos niveles de desarrollo del curriculum escolar.

Si bien durante todo el tiempo hemos hablado de aplicar el programa en la escuela, creemos que este tipo de experiencias pueden también ser llevadas a cabo en contextos extraescolares como grupos de tiempo libre, o grupos de terapia infantojuvenil, realizando algunas adaptaciones necesarias en función del grupo con el que se quiera realizar la intervención.

\section{Referencias}

BLURTON-JONES, N. (1967). An ethological study of some aspects of social behavior of children in nursery school. En D. Morris (Ed). Primate Ethology. Weidenfeld and Nicolson.

BRUner, J. (1986). Juego, pensamiento y lenguaje. Perspectivas, 16 (1), 79-85.

BUTLER, R. (1989). Interest in the task and interest peer' work in competitive and non competitive conditions: A Development Study. Child Development, 60 (3), 562-570.

Cascón, P., y MARTín, C. (1986). La alternativa del juego: Fichas térnicas. Torrelavega: Colectivo Educadores para la Paz.

Coll, C. (1984). Estructura grupal, interacción entre alumnos y aprendizaje escolar. Infancia y Aprendizaje, 27-28, 119-139.

De LA CRUZ, V. (1982). Batería de aptitudes para el aprendizaje escolar. TEA. Madrid.

De la Cruz, V., y MaZaira, M. C. (1992). DSA. Programa de desarrollo socio-afectivo. Madrid: TEA Ediciones.

DeuTsCH, M. (1971). Efectos de la cooperación y la competición sobre el proceso de grupo. En D. Cartwright y A. Zarder (Eds). Dinámica de grupo (pp. 503-525). México: Trillas.

ElKonin, D. B. (1980). Psicología del juego. Madrid: Pablo del Río (Trabajo original publicado en 1978).

FISHER, E. P. (1992). The impact of play on development: A meta-analysis. Play \& Culture, 5 (2), 159-181.

Forman, E., y Cazden, C. (1984). Perspectivas vygotskianas en la educación. El valor cognitivo de la interacción entre iguales. Infancia y Aprendizaje, 27-28, 139-157.

GaraigordobiL, M. (1990). Juego y desarrollo infantil. Madrid: Seco-Olea.

Garaigordobil, M. (1992a). Diseño y evaluación de un programa lúdico de intervención psicoeducativa con niños de 6-7 años. Servicio Editorial de la Universidad del País Vasco (Microfichas de Tesis Doctorales).

GaRAIGORDOBIL, M. (1992b). Juego cooperativo y socialización en el aula. Madrid:Seco-Olea.

GARAIGORDOBIL, M. (1993). Un estudio correlacional de las vinculaciones entre la conducta social con otras variables socio-cognitivas y afectivas del desarrollo infantil. Revista de Psicología de la Educación, 11, 49-71.

Hall, R.; Rocklin, T.; Dansereau, D.; Skaggs, L.; O'Donell, A.; Lambiotte, J., y Young, M. (1988). The role of individual differences in the cooperative learning of technical material. Journal of Educational psychology, 80 (2), 172-178.

Hoffman, M. L. (1983). Desarrollo Moral y Conducta. En M.R. Schaffer (Ed). El mundo social del niño. Madrid: Aprendizaje Visor.

JoHNSON, D. W., y JOHNSON, R. T. (1986). Impact of classroom organization and instructional methods on the effectiveness of mainstreaming. En J. Meisel (Ed). Mainstreaming handicapped children: Outcomes, controversies and new directions (pp. 219-250). Hillsdale: NJ: Erlbaum. 
Kamil, C., y Devries, R. (1988). Juegos colectivos en la primera enseñanza. Implicaciones de la teoría de Piaget. Madrid: Aprendizaje Visor. (Trabajo original publicado en 1980).

Kohlberg, L. (1982). Estadios Morales y Moralización. El enfoque cognitivo - evolutivo. Infancia y Aprendizaje, 18, 33-51.

KOPPITZ, E. (1974). El Dibujo de la Figura Humana en los niños. Buenos Aires: Guadalupe.

LEVY, A.; WOLFGANG, CH., y KOORLAND, M. (1992). Sociodramatic play as a method for enhancing the language performance of kindergarten age students. Early Childhood Research Quarterly, 7 (2), 245-262.

MCCUNE-NICOLICH, L. (1981). Towards symbolic functioning: structure of early pretend games and potential parallels with languaje. Child Development, 52, 785-797.

MARINO, R. F. (1992). The relationship of pretense play, sociability and language development, in three to five-year old children. Dissertation Abstracts International, 53 (5-A), 1455.

MARTINEK, T., y ZAICHKOWSKY, L. (1977). Martinek \& Zaichkousky:self concept scale for children. Psychologist and Educators, inc. Jaksonville.

MARTÍNEZ, M. (1986). Juegos cooperativos, una nueva forma de jugar. Cuadernos de Pedagogía, 140 , 19-23

MASNOU, F. (1991). Educació per a la convivéncia. Barcelona: EUMO.

MENDER, J.; KERR, R., y ORLICK, T. (1982). A cooperative games program for learning disabled children. International Journal of Sport Psychology, 13 (4), 222-233.

Mujina, V. (1975). Psicología de la edad preescolar. Madrid: Pablo del Río.

ORLICK, T. (1986). The cooperative sports and games book. (Juegos y deportes cooperativos. Madrid: Popular. (Trabajo original publicado en 1978).

OrLICK, T. (1981). Positive socialization via cooperative games, Developmental Psychology, 17 (4), 426-429

Orlick, T. (1988). El juego cooperativo. Cuadernos de Pedagogía, 163, 84-87.

Ortega, R. (1986). Juego y pensamiento en los niños. Cuadernos de Pedagogía, 133, 33-35.

ORTEGA, R. (1987). El juego: Un laboratorio de comunicación social. Actas de las V Jornadas de Estudio sobre la Investigación en la Escuela. Sevilla.

Ortega, R. (1991a). El juego sociodramático y el desarrollo de la comprensión y el aprendizaje social. Infancia y Aprendizaje, 55, 87-121.

ORTEGA, R. (1991b). Relaciones afectivas, comprensión social y juego dramático en la educación escolar. Investigación en la escuela, 15, 29-44.

Ortíz, M. J.; Apodaca, P.; Echevarrfa, I.; Eceiza, A.; Fuentes, M. J., y López, F. (1991). Propuesta de intervención educativa para el desarrollo de la conducta prosocial altruista. Actas del Cogreso de Intervención Educativa. Madrid.

OVEJERO, A. (1990). El aprendizaje cooperativo como alternativa a la enseñanza tradicional. Barcelona: PPV.

Ovejero, A. (1993). Aprendizaje cooperativo: Una eficaz aportación de la psicología social a la escuela del siglo XXI, Psicothema, 5, 373-390.

Pardos, M. L.; Gómez, C.; Miguel, A., y Bernard, M. C. (1988). Posibilidades educativas del juego dramático. Revista Internacional de Formación de Profesorado, 2, 275-280.

Pellegrini, A. D., y Galda, L. (1993). Ten years after: A reexamination of simbolic play and literacy research. Reading Research Quarterly, 28 (2), 163-175.

Piaget, J. (1974). El juicio moral en el niño. Barcelona: Fontanella. (Trabajo original publicado en 1932).

PiageT, J. (1979). La formación del símbolo en el niño. México: Fondo de cultura económica. (Trabajo original publicado en 1959).

Piaget, L., e INHelder, H. (1984). La psicología del niño. Madrid: Morata. (Trabajo original publicado en 1969).

RuE, J. (1989). El trabajo cooperativo por grupos. Cuadernos de Pedagogía, 170, 18-21.

SHERIF, M.; HARVEY, O. Y.; WhITE, B. Y.; HOOD, W. R., y SHER IF C. W. (1961). Intergroup Conflict and Cooperation: the Robbers Cave Experiment. Norman, Oklahoma: University of Oklahoma.

Silva, F., y MARTORELl, C. (1983). BAS 1 y 2. Batería de socialización. Madrid: TEA.

SLAVIN, R. (1983). When does cooperative learning increase scudent achievement? Psychological Bulletin, 94, 429-445.

SLAvIN, R. (1990). Cooperative learning: Theory, research and practice. Englewood Cliffs: NJ: PrenticeHall.

STERLING, A. (1990). Social behavior updated: A review of Eisenberg \& Mussen's the roots of prosocial behavior in children. Merrill-Palmer Quarterly, 36(4), 581-584.

VYGOTSKY, L. S. (1982). El juego y su función en el desarrollo psíquico del niño. Versión Castellana de la conferencia dada por Vygotski en el Instituto Pedagógico Estatal de Hertzsn. Leningrado. En R. Grasa. Cuadernos de Pedagogía, 85, 39-49. (Trabajo original publicado en 1933).

VYGOTSKY, L. S. (1979). El desarrollo de los procesos psicológicos superiores. Barcelona: Crítica Grijalbo. (Trabajo original publicado en 1934)

WINNICOTT, D. (1982). Realidad y juego. Barcelona: Gedisa. (Trabajo original publicado en 1971).

ZaBALZA, M. (1987). Didáctica de la educación infantil. Tomo I-II. Madrid: Narcea. 


\section{Bibliografía básica. Sugerencia para los lectores}

GARAIGORDOBIL, M. (1992a). Diseño y evaluación de un programa lúdico de intervención psicoeducativa con niños de 6-7 años. Servicio Editorial de la Universidad del País Vasco (Microfichas de Tesis Doctorales).

GARAIGORDOBLL, M. (1992b). Juego cooperativo y socialización en el aula. Madrid:Seco-Olea.

ORLICK, T. (1986). The cooperative sports and games book. (Juegos y deportes cooperativos. Madrid: Popular. (Trabajo original publicado en 1978)

Zabalza, M. (1987). Didáctica de la educación infantil. Tomo I.II. Madrid: Narcea.

\section{Una metodología para la utilización didáctica del juego en contextos educativos Maite Garaigordobil CL\&E, 1995, 25, pp. 91-105}

Resumen: En este trabajo se expone la didáctica aplicada de un programa de juego, que tiene por finalidad estimular el desarrollo social de niños de 6 a 8 años. El articulo analiza el valor del juego y de la interacción cooperativa entre iguales en el desarrollo infantil, describiendo el programa y su didáctica, que ha sido sistematizada en el trascurso de 4 años de aplicación en diversas aulas de ciclo inicial. Esta intervención psicoeducativa ha sido validada experimentalmente mediante una investigación de diseño pretest-intervención-postest, cuyos resultados se describen brevemente remitiendo al lector a trabajos previos donde han sido publicados (Garaigordobil, 1992 a.b; 1993). La didáctica diseñada y propuesta en esta intervención psicoeducativa es generalizable a la aplicación de programas de juego psicomotriz, creativo... para estimular otras dimensiones del desarrollo infantil desde contextos educativos.

Datos sobre la autora: Maite Garaigordobil es Doctora en Psicología y profesora de Psicodiagnóstico en la Facultad de Psicología de la Universidad del País Vasco. Actualmente trabaja en el estudio del juego infantil, diseñando y evaluando programas lúdicos de intervención psicoeducativa. Es autora de dos obras (Juego y desarrollo infantil, Madrid, SecoOlea, 1990; Juego cooperativo y socialización en el aula, Madrid, Seco-Olea, 1992) en las que se analiza el papel del juego en el desarrollo y su utilización didáctica. En los últimos años, una parte de su actividad ha estado centrada en el diseño y evaluación de programas lúdicos de intervención psicoeducativa que estimulan distintos procesos del desarrollo infantil. En la actualidad, siguiendo esta línea de investigación que presenta en el articulo, ha diseñado un nuevo programa con 55 actividades para promover la comunicación, la socialización y la conducta altruista en niños de 8 a 10 años. Este programa está siendo implementado en 4 aulas experimentales y al finalizar el curso académico evaluaremos sus efectos pretest-postest en la conducta social aserriva, en los mensajes que se dan en la comunicación intragrupo, en las conductas de ayuda dentro del aula, en la conducta altruista con los compañeros, y en el autoconcepto. Por otro lado, recientemente escá realizando estudios pilotos con algunas técnicas para evaluar e intervenir en la creatividad infantil.

Dirección: Facultad de Psicología. Dpto. Personalidad, Evaluación y Tratamientos. Universidad del País Vasco. Avda de Tolosa 70. San Sebastian. 20.009. Guipuzcoa.

(C) PERMISOS PARA CITAR O REPRODUCIR EN OTRAS FUENTES: Se pueden citar libremente hasta 500 palabras. Para reproducir una porción de texto mayor, figuras o ilustraciones, se deberá pedir permiso por escrito a la revista, especificando el uso al que se destina el texto. En todos los casos, se deberá citar el copyright de $C L \& E$. En el caso de artículos o textos que hayan sido a su vez reproducidos en $C L \& E$ los interesados deberán dirigirse tanto a los detentadores del copyright original como a $C L \& E$, en el caso de que se quiera hacer uso de la traducción. FOTOCOPIAS: Para todo lo relacionado con el uso mediante fotocopia del material de esta revista, deberán dirigirse a: CEDRO, C/ José Marañón, 10, 3. Izda. Tel. 5941575 . Fax 4453567 